\title{
Influence of local lithium metal deposition in 3D microstructures on local and global behavior of Lithium-ion batteries
}

\author{
Simon Hein ${ }^{\mathrm{a}, \mathrm{b}, \mathrm{c}, 1, *}$, Arnulf Latz $\mathrm{L}^{\mathrm{a}, \mathrm{b}, \mathrm{c}, 1}$ \\ ${ }^{a}$ German Aerospace Center (DLR), Institute of Engineering Thermodynamics,Pfaffenwaldring 38-40,70569 Stuttgart, Germany \\ ${ }^{b}$ Helmholtz Institute for Electrochemical Energy Storage (HIU), Helmholtzstrasse 11, 89081 Ulm, Germany \\ ${ }^{c}$ University of Ulm (UUlm), Institute of Electrochemistry, Albert Einstein-Allee 47, 89081 Ulm, Germany
}

\begin{abstract}
On of the major degradation processes in lithium ion batteries is the deposition of metallic lithium on the surface of the active particles in the negative electrode. In this paper we present a fully 3D microstructure resolved simulation of the influence of plated lithium on the cell potential during discharge depending on the amount and position of plated lithium. Our simulations give insight on the most probable position of the first occurrence of plating within the electrode depending on applied current and ambient temperatures as well as on the subtle local electric current distributions during stripping of plated lithium upon discharge. Specifically a stripping induced intercalation of lithium ions in the supporting graphite material during discharge is discovered. This phenomenon, easily accessible to microstructure resolved simulations, leads to a violation of the relation between transferred charge during stripping and the amount of plated lithium. As a consequence the amount of plated lithium cannot be uniquely determined from the applied current and the length of the potential plateau during stripping. We show that the value and length of the plateau depends on the amount of plated lithium, the fraction of the surface area covered by lithium and the applied current.
\end{abstract}

Keywords: lithium plating, lithium stripping, 3D microstructure, modeling and simulation, theory

\section{Introduction}

One of the major safety risks of lithium-ion batteries is the occurrence of an electrical contact between the two opposing electrodes, which potentially can lead to a catastrophic thermal runaway as in the dreamliner event [1] or at least to a highly unbalanced battery. The deposition of metallic lithium on the surface of the anode particles together with the initiation of dendritic growth of lithium is the most probable cause for such a short 10 circuit [2]. Instead of being intercalated in the anode particles, the lithium begins under certain process and environmental conditions to accumulate on the surface of the anode. Because of the similarity to electrodeposition [3], this process in lithium-ion batteries is named ${ }_{15}$ lithium plating. The resulting metallic phase poses a huge safety hazard and can significantly reduce the cell durability and cycleability [4-7]. Various methods to

\footnotetext{
${ }^{*}$ Corresponding author:

Email address: Simon. Hein@DLR.de (Simon Hein)

URL: http://www.dlr.de/tt/en/ (Simon Hein)

${ }^{1}$ ISE member
}

identify plated lithium were proposed using in-situ, exsitu or in-operandi measurement methods. The major ${ }_{20}$ problem of these methods is, that they can either only detect plated lithium indirectly (during discharge [8, 9]) or with setups not applicable outside of a research environment (SEM [10], optical microscope [11], neutron diffraction [12]). Theoretical investigations and simulations are hence of growing importance.

Lithium plating is so far mostly modeled in the frame of porous electrode theory [7, 13,-16] or on the base of equivalent circuits [17]. In these two type of models the microstructure is only represented by morphological paso rameters as e.g. average porosity and tortuosity. Porous electrode theory additionally accounts for the diffusion in some representative particle for each volume element. Inhomogeneities on electrode scale can theoretically enter in the form of spatially dependent morpho35 logical parameter, but are usually neglected. Modifications like this might be important to investigate experimentally observed non-uniform plated lithium distribution in large pouch cells [18]. The role of the always present local fluctuation of the electrochemical 

the electrode cannot be resolved within this approach. Especially the appearance of a metallic film on single particles, which drastically changes the electrochemical situation locally and may lead to a potentially self accelerating phenomenon is only accessible to a fully 3D microstructure resolved modelling approach.

So far, spatially resolved Lithium plating as e.g. observed in [11] inside a 3D microstructure have not been investigated theoretically. Models of dendritic lithium 50 deposition, only resolve single dendrites usually in axially symmetric approximation [19, 20]. In our paper a first step towards the construction of a microstructure resolved $3 \mathrm{D}$ plating model is taken. The approach is split into two different parts. First we study the influ55 ence of operating conditions on the spatial distribution of the onset of plating facilitating full-cell simulations for different operating conditions. Then, the influence of spatially distributed metallic lithium in a graphite anode on the delithiation characteristics of a lithium ion 60 half-cell is investigated in detail. In the literature [9] the usually observed stripping plateau in the cell potential is used to estimate the amount of plated lithium. With our explicit simulation of the stripping process on microstructure scale it is possible to test the as-

65 sumptions underlying the experimental investigations. Specifically, we show that the length of the plateau not only depends on the amount of plated lithium but also on its spatial distribution and the detailed microstructure of the anode, on which lithium is deposited.

\section{2. Theory}

\subsection{Lithium intercalation battery - Thermodynamic consistent transport model}

In general a lithium intercalation battery consists of three porous phases: a negative electrode, a separa75 tor and a positive electrode. The pore space of these materials is filled with an electrolyte. In Fig. 1 the microstructure of the lithium ion battery used in this work is shown. The lithium ions are stored inside the solid phase of anode and cathode. During charg-

$80 \mathrm{ing} /$ discharging operation the lithium is deintercalated from the cathode/anode, transported through the electrolyte inside the pore space and then intercalated into the anode/cathode. The anode - cathode or through plane direction (x-direction in our simulation) repre85 sents the full cell thickness and is resolved in all structural details. In lateral directions, insulating boundary conditions are applied i.e neither electrical current nor ions can leave the simulation domain in these directions. This boundary condition is an approximation and

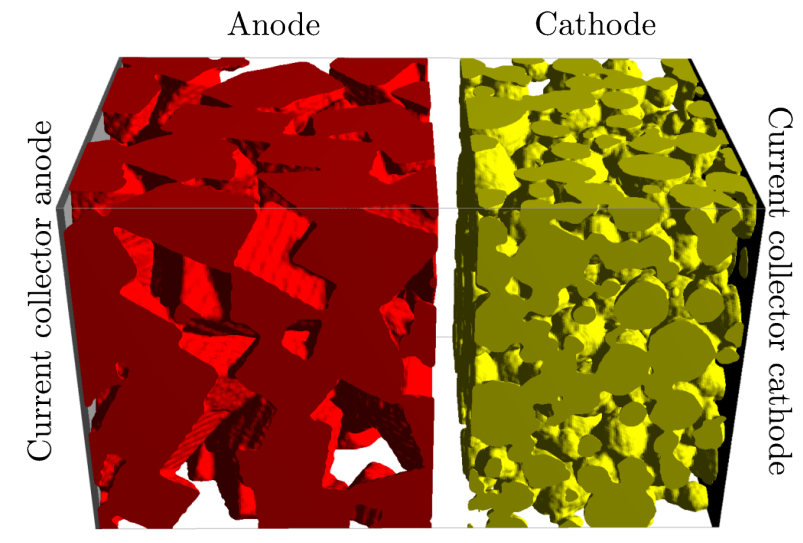

Figure 1: Full cell microstructure of a lithium ion battery: The anode (left/red) consists of graphite and the cathode (right/yellow) consists of lithium manganese oxide (LMO). The separator is included as an effective porous medium between the two electrodes. The anode is in electrical contact with the negative current collector (far left/grey) and the cathode with the positive current collector (far right/black).

90 neglects possible in-plane currents in lateral directions at the boundary of the simulation domain. Due to the statistical isotropy of the electrodes and the main potential drop in trough plane direction, this approximation is justified. Artificial boundary effects due this boundary condition are not observed in our simulation. The use of periodic boundary conditions is not possible since the cathode is a cut out of a larger structure and thus not periodic. The electrical connection between the battery and an external circuit is achieved by applied specific boundary conditions to the current collectors. The negative current collector has a constant potential as boundary condition, while the positive current collector has a constant current flux for charging or discharging. An applied constant potential is represented by setting the cathode current collector to the desired cell potential plus the potential of the negative current collector.

\subsubsection{Basic transport model}

The states of the lithium-ions inside the different phases of the battery are described by the concentration in the solid $\left(c_{\mathrm{So}}\right)$ and in the electrolyte $\left(c_{\mathrm{El}}\right)$ and by the electrical potential in the solid $\left(\Phi_{\text {So }}\right)$ and the electrochemical potential in electrolyte $\left(\varphi_{\mathrm{El}}\right)$. The time evolution of these variables is obtained by solving the mass balance equation and charge conservation equation in the different phases. These equations are given by Eq. 
(11).

$$
\begin{array}{rlrl}
\partial_{t} c_{\mathrm{El}} & =-\nabla N_{\mathrm{El}} & \partial_{t} c_{\mathrm{So}} & =-\boldsymbol{\nabla} \boldsymbol{N}_{\mathrm{So}} \\
0 & =-\nabla j_{\mathrm{El}} & 0 & =-\boldsymbol{\nabla} \boldsymbol{j}_{\mathrm{So}}
\end{array}
$$

where $N_{\mathrm{i}}$ is the particle flux in phase $i$ :

$$
\begin{aligned}
& N_{\mathrm{El}}=-D_{\mathrm{El}} \nabla c_{\mathrm{El}}+\frac{t_{+}}{F} j_{\mathrm{El}} \\
& N_{\text {So }}=-D_{\mathrm{So}} \nabla c_{\text {So }}
\end{aligned}
$$

and $j_{\mathrm{i}}$ the current density in phase $i$ :

$$
\begin{aligned}
& \boldsymbol{j}_{\mathrm{El}}=-\kappa_{\mathrm{El}} \nabla \varphi_{\mathrm{El}}+\kappa_{\mathrm{El}} \frac{1-t_{+}}{F}\left(\frac{\partial \mu_{\mathrm{El}}}{\partial c_{\mathrm{El}}}\right) \nabla c_{\mathrm{El}} \\
& \boldsymbol{j}_{\mathrm{So}}=-\sigma_{\mathrm{So}} \boldsymbol{\nabla} \Phi_{\mathrm{So}}
\end{aligned}
$$

The two phases $i$ are either solid (So) or electrolyte (El). Different materials (e.g. Graphite, $\mathrm{LiPF}_{6}$ in $\mathrm{PC} / \mathrm{EC} / \mathrm{DMC}, \ldots)$ are represented by their respective parameters (Diffusion coefficient $D_{\mathrm{i}}$, electrical/ionic conductivity $\sigma_{\mathrm{i}} / \kappa_{\mathrm{i}}$, transference number $t_{+}$and open circuit potential (OCP) $U_{0}$ ).

The equations of the different phases are coupled by interface conditions at their respective interface:

$$
\begin{array}{rr}
\boldsymbol{j}_{\mathrm{El}} \cdot \boldsymbol{n}_{\mathrm{So}-\mathrm{El}}=i_{\mathrm{SoEl}} & \boldsymbol{j}_{\mathrm{So}} \cdot \boldsymbol{n}_{\mathrm{So}-\mathrm{El}}=i_{\mathrm{SoEl}} \\
\boldsymbol{N}_{\mathrm{El}} \cdot \boldsymbol{n}_{\mathrm{So}-\mathrm{El}}=\frac{i_{\mathrm{SoEl}}}{F} & \boldsymbol{N}_{\mathrm{So}} \cdot \boldsymbol{n}_{\mathrm{So}-\mathrm{El}}=\frac{i_{\mathrm{SoEl}}}{F}
\end{array}
$$

with the area normal $\boldsymbol{n}_{\text {So-El }}$ pointing from the solid into the electrolyte and $i_{\text {SoEl }}$ the interface current between the solid and the electrolyte phase. A symmetric ButlerVolmer-equation can be used to describe the intercalation reaction,

$i_{\mathrm{SoEl}}=2 \cdot i_{00} \sqrt{c_{\mathrm{El}} c_{\mathrm{So}}\left(c_{\mathrm{So}}^{\max }-c_{\mathrm{So}}\right)} \sinh \left(\frac{F}{2 R T} \cdot \eta_{\mathrm{SoEl}}\right)$

Without additional side reactions, only anodeelectrolyte (An-El) and cathode-electrolyte (Ca-El) interfaces have to be considered. The interface currents $i_{\mathrm{An}-\mathrm{El}}$ and $i_{\mathrm{Ca}-\mathrm{El}}$ are modeled as symmetric ButlerVolmer-equation (Eq. (5)) with different overpotentials due to their different OCP and different kinetic properties. For more detailed information and explanations about the underlying theory the reader is referred to [21, 22].

\subsection{Reactions}

To quantify the kinetics the proper overpotentials $\eta$

\begin{tabular}{|c|c|c|}
\hline \multicolumn{3}{|c|}{ For species $\mathrm{i}$ in phase $\mathrm{p}$} \\
\hline w.r.t.a.r. & $\mu_{\mathrm{i}}^{\mathrm{p}}=\mu_{\mathrm{i}}^{\mathrm{p}, \Theta}+R T \log \left(a_{\mathrm{i}}^{\mathrm{p}}\right)$ & \multirow[b]{3}{*}{ (6) } \\
\hline w.r.t.a.r. & $\tilde{\mu}_{\mathrm{i}}^{\mathrm{p}}=z_{\mathrm{i}} F \Phi_{\mathrm{p}}+\mu_{\mathrm{i}}^{\mathrm{p}, \Theta}+R T \log \left(a_{\mathrm{i}}^{\mathrm{p}}\right)$ & \\
\hline w.r.t.a.r. & $\tilde{\mu}_{\mathrm{i}}^{\mathrm{p}}=z_{\mathrm{i}} F \Phi_{\mathrm{p}}+\mu_{\mathrm{i}}^{\mathrm{p}}$ & \\
\hline \multicolumn{3}{|c|}{ Lithium ions in electrolyte } \\
\hline w.r.t.a.r. & $\tilde{\mu}_{\mathrm{Li}^{+}}^{\mathrm{El}}=F \Phi_{\mathrm{El}}+\mu_{\mathrm{Li}^{+}}^{\mathrm{El}}$ & (7) \\
\hline w.r.t.l. & $\varphi_{\mathrm{Li}^{+}}^{\mathrm{El}}=\Phi_{\mathrm{El}}-\frac{\mu_{\mathrm{Li}^{\mathrm{Li}}}-\mu_{\mathrm{Li}^{+}}^{\mathrm{Li}}}{F}$ & (8) \\
\hline \multicolumn{3}{|c|}{ Lithium ions in solid } \\
\hline w.r.t.a.r. & $\tilde{\mu}_{\mathrm{Li}^{+}}^{\mathrm{So}}=F \Phi_{\mathrm{So}}+\mu_{\mathrm{Li}^{+}}^{\mathrm{So}}$ & (9) \\
\hline w.r.t.l. & $\varphi_{\mathrm{Li}^{+}}^{\mathrm{So}}=\Phi_{\mathrm{So}}-U_{0}$, with $U_{0}=\frac{\mu_{\mathrm{Li}}^{\mathrm{ref}}-\mu_{\mathrm{Li}^{+}}^{\mathrm{So}}}{F}$ & $(10)$ \\
\hline
\end{tabular}
have to calculated. If side reactions as plating are added
Table 1: The definitions of the electrochemical potential of the different involved species.

$\Phi_{\mathrm{p}}$ electrical potential in phase $\mathrm{p}$

$\mu_{\mathrm{i}}^{\mathrm{P}}$ chemical potential of species $\mathrm{i}$ in phase $\mathrm{p}$

$\tilde{\mu}_{\mathrm{i}}^{\mathrm{p}}$ electrochemical potential of species $\mathrm{i}$ in phase $\mathrm{p}$ (w.r.t.a.r.)

$\varphi_{\mathrm{i}}^{\mathrm{p}}$ electrochemical potential of species $\mathrm{i}$ in phase $\mathrm{p}$ (w.r.t.l.)

not only solid - electrolyte overpotentials $\eta_{S o-E l}$ as in Eq. 5 for intercalation and plating but also solid -solid overpotentials for lithium transfer from plated lithium to intercalated lithium have to be considered. The two intercalation reactions in a lithium-ion battery can be written the following way:

$$
\begin{aligned}
& \mathrm{Li}_{\mathrm{El}}^{+} \rightleftharpoons \mathrm{Li}_{\mathrm{An}}^{+} \\
& \mathrm{Li}_{\mathrm{El}}^{+} \rightleftharpoons \mathrm{Li}_{\mathrm{Ca}}^{+}
\end{aligned}
$$

The two different side reactions at the anode, which we will describe are lithium deposition from the electrolyte onto the surface of the graphite (lithium plating) and chemical intercalation of plated lithium. Since we first want to develop the theory for reversible plating, the formation of a solid-electrolyte-interphase will be neglected.

$$
\begin{aligned}
\mathrm{Li}_{\mathrm{El}}^{+}+e^{-} & \rightleftharpoons \mathrm{Li}_{\mathrm{Pl}}^{0} \\
\mathrm{Li}_{\mathrm{Pl}}^{0} & \rightarrow \mathrm{Li}_{\mathrm{So}}^{0}
\end{aligned}
$$

with the index So representing graphite.

\subsubsection{Intercalation}

The overpotential of the intercalation reaction into graphite (I-A) is given by

$$
F \cdot \eta_{\text {intercalation }}=\tilde{\mu}_{\mathrm{Li}^{+}}^{\mathrm{So}}-\tilde{\mu}_{\mathrm{Li}^{+}}^{\mathrm{El}}
$$


By changing the reference to lithium and with Eq. 8) and (10), we can rewrite it to

$$
\begin{aligned}
F \cdot \eta_{\text {intercalation }} & =\tilde{\mu}_{\mathrm{Li}^{+}}^{\mathrm{So}}-\tilde{\mu}_{\mathrm{Li}}^{\mathrm{ref}}+\tilde{\mu}_{\mathrm{Li}}^{\mathrm{ref}}-\tilde{\mu}_{\mathrm{Li}^{+}}^{\mathrm{El}} \\
\eta_{\text {intercalation }} & =\Phi_{\mathrm{So}}-U_{0}-\varphi_{\mathrm{El}}^{\mathrm{Li}^{+}}
\end{aligned}
$$

A symmetric Butler-Volmer relation is used for the exchange-current density:

$$
i_{\text {intercalation }}=2 \cdot i_{\text {intercalation }}^{0} \cdot \sinh \left(\beta \cdot \eta_{\text {intercalation }}\right)
$$

with $i_{\text {intercalation }}^{0}=i_{\text {inter }}^{00} \sqrt{c_{\mathrm{El}} c_{\mathrm{So}}\left(c_{\mathrm{So}}^{\max }-c_{\mathrm{So}}\right)}$ and $\beta=$ $F /(2 R T)$

\subsubsection{Lithium Plating}

During lithium plating a metallic film is deposited on top of the surface of an electrode. The plating (deposition) and stripping (dissolution) of lithium is described by the reaction in Eq. (S1). The reaction is thermodynamically possible if the difference in the electrochemical potentials becomes negative. With $\mu_{L i^{0}}^{P l}=\mu_{L i^{+}}^{P l}+\mu_{e^{-}}^{P l}$, the overpotential of the deposition can be written as

$$
F \cdot \eta_{\text {plating }}=\tilde{\mu}_{\mathrm{Li}^{+}}^{\mathrm{Pl}}-\tilde{\mu}_{\mathrm{Li}^{+}}^{\mathrm{El}}
$$

With the definition of the electrochemical potentials (Eq. (8) and Eq. (9)), we obtain

$$
\eta_{\text {plating }}=\Phi_{\mathrm{Pl}}-\varphi_{\mathrm{Li}^{+}}^{\mathrm{El}}
$$

We assume, that the electrical potential at the interface between plated lithium and graphite is continuous. If the plating overpotential is below zero $\left(\eta_{\text {plating }}<0\right)$, lithium from the electrolyte can be deposited on the surface. Conversely, lithium dissolution/stripping takes place, if the overpotential reaches a positive value. From this point on, we call this the "plating condition".

$$
\Phi_{\mathrm{Pl}}-\varphi_{\mathrm{Li}^{+}}^{\mathrm{El}}<0
$$

Assuming, that the lithium phase can not be fully depleted, a Butler-Volmer-like expression can be used for the exchange-current density:

$$
i_{\text {plating }}=i_{\text {plating }}^{0}\left(\exp \left(\beta \eta_{\text {plating }}\right)-\exp \left(-\beta \eta_{\text {plating }}\right)\right)
$$

Since lithium metal has a higher conductivity than graphite, we can approximate the electrical potential $\Phi_{\mathrm{Pl}}$ in the plated lithium by the electrical potential in the graphite $\Phi_{\text {So }}$. Hence the plating condition can equivalently be expressed by the intercalation overpotential $\eta_{\text {intercalation }}$ and the open circuit potential $U_{0}\left(c_{\mathrm{So}}\right)$

$$
\eta_{\text {intercalation }}+U_{0}<0
$$

\subsubsection{Reactions of plated lithium}

Plated metallic lithium on the electrode particle is not stable even if no external current is applied. The overpotential of the chemical intercalation can be written as

$$
F \cdot \eta_{\mathrm{S} 2}=\tilde{\mu}_{\mathrm{Li}}^{\text {Anode }}-\tilde{\mu}_{\mathrm{Li}}^{\text {Plated }}
$$

Since the lithium metal is in direct contact with the anode material, their Fermi energies will equilibrate. As a consequence it can be seen that the overpotential for the chemical intercalation of lithium is the same as the open circuit potential between anode material and metallic lithium 2 i.e.

$$
\eta_{\mathrm{S} 2}=-U_{0}
$$

As long as the open-circuit potential $U_{0}$ is positive, plated lithium will always be intercalated into the supporting graphite.

\subsubsection{Reversible stripping of plated lithium}

The deposited metallic lithium can be reversibly reutilized for battery operations in two ways. Either the deposited lithium is stripped upon discharge of the battery (S1) and then intercalated into the anode (I-A) or cathode material (I-C), or the cell is stored under open circuit condition until the metallic lithium is 140 electro-neutrally intercalated in the supporting anode (S2). These two pathes are not equivalent under nonequilibrium conditions, even though the final states are thermodynamically, i.e. in equilibrium, identical.

The direct chemical intercalation Eq. $(\mathrm{S} 2)$ is a process independent from the electrical potential in the anode phase (Eq. (21)). Hence the changes in the cell potential are purely due to the increase of the state of charge of the anode.

The electrochemical process of lithium stripping (Eq. (S1) has a equilibrium potential vs a lithium reference of zero. Thus a anode with plated lithium exhibits a mixed potential during combined stripping and delithiation, which has a value between the cell potential of pure anode-delithiation and pure lithium stripping. While a stripping current is applied to a plated lithium-ion battery, the newly stripped lithium ion has a chemical potential which makes it favorable to intercalate as well in the anode as the cathode material. Therefore instead of being transported to the cathode before intercalation

\footnotetext{
${ }^{2}$ In the definition of the OCP the roles of Fermi energies and chemical potential of lithium ions are reversed: The electrochemical potentials of $\mathrm{Li}$ ions are in equilibrium and the difference of the chemical potentials is related to the difference of the Fermi energies and thus the measured OCP
} 
it can also be directly re-intercalated into the anode. The intercalation into the anode material will result in a globally electro-neutral process, since the electrons freed in the anode during lithium dissolution are consumed during intercalation in the anode. The energy barriers to be overcome and thus the overpotentials and the dependence on the applied current will be different than in the direct chemical intercalation.

\section{Simulation}

All simulations in this work are based on the framework BEST (Battery and Electrochemistry Simulation Tool [23]), which is a finite volume implementation 180 of the thermodynamic transport model, derived in [21] (See Sec. 2.1). The implementation was extended by our new model for the additional lithium phase (Eq. (16) and $(18 / 22)$ ). The simulations are run on the high performance computing cluster JUSTUS, located at the university of Ulm with parallelization on 8 cores. All simulations are done isothermal.

\subsection{Numerical approximations}

A new numerical challenge of the stripping simulation is the vanishing of the lithium phase. The ButlerVolmer and similar interface current expressions are derived under the assumptions of non-depleting phases. The expression in Eq. (18) is only valid, if the dissolution of the lithium phase does not lead to a vanishing of the phase. Additional assumptions are necessary if one of the phases involved in the electrochemical exchange is consumed during the electrochemical or chemical reaction. In this work, we approximate the behavior of a vanishing phase by modifying that part of the interface current, which is responsible for the dissolution of lithium (for $i_{L i / L i^{+}}>0$ ). The expression for the exchange current between the lithium metal and the electrolyte is set to be:

$$
\begin{aligned}
i_{\text {plating }} & =i_{\text {plating }}^{0} \cdot g\left(\eta_{\text {plating }}, d_{\text {Li }}\right) \\
g\left(\eta_{\text {plating }}, d_{\mathrm{Li}}\right) & =f\left(d_{\mathrm{Li}}, d_{0}\right) \cdot \exp \left(\beta \cdot \eta_{\text {plating }}\right) \\
& -\exp \left(-\beta \cdot \eta_{\text {plating }}\right)
\end{aligned}
$$

with the overpotential of the reaction from Eq. (16), the current prefactor $i_{\text {plating }}^{0}=i_{\text {plating }}^{00} \cdot \sqrt{c_{\mathrm{El}}}$ and $\beta=F / 2 R T$. The current thickness of the plated lithium is described by $d_{L i}$. The dissolution of plated lithium stops, if the thickness of the plated lithium reaches zero and a further dissolution is physically impossible. This fact is represented by the prefactor $f\left(d_{\mathrm{Li}}, d_{0}\right)$, which turns off lithium dissolution below an artificially chosen thickness $d_{0}$. The obvious approach is the usage of the Heaviside function. Adopting this function introduces a singularity into the equation system. If the lithium thickness $d_{L i}$ vanishes while solving the equation system, numerical instabilities occur, which can prevent a further computation. A regularization technique of singularities in source terms is applied to prevent or at least minimize the influence on the numerical stability. [24] A numerically stable choice was found to be:

$$
f\left(d, d_{0}\right)=\frac{d^{4}}{d_{0}^{4}+d^{4}}
$$

The influence of the exact value of $d_{0}$ was investigated by comparing the simulation results for four different values $(0.48 \mathrm{~nm}, 0.24 \mathrm{~nm}, 0.048 \mathrm{~nm}$ and $0.0048 \mathrm{~nm}$ ). The thickness of a lithium metal monolayer would be $0.28 \mathrm{~nm}$. The choice of smaller value for $d_{0}$ still has meaning as a numerical approximation for the Heaviside function (jump from one to zero at the monolayer thickness). In Fig. 2 the cell potential during a stripping simulation (no chemical intercalation enabled) with applied constant current as function of time for the four different prefactors is shown. It can bee seen, that

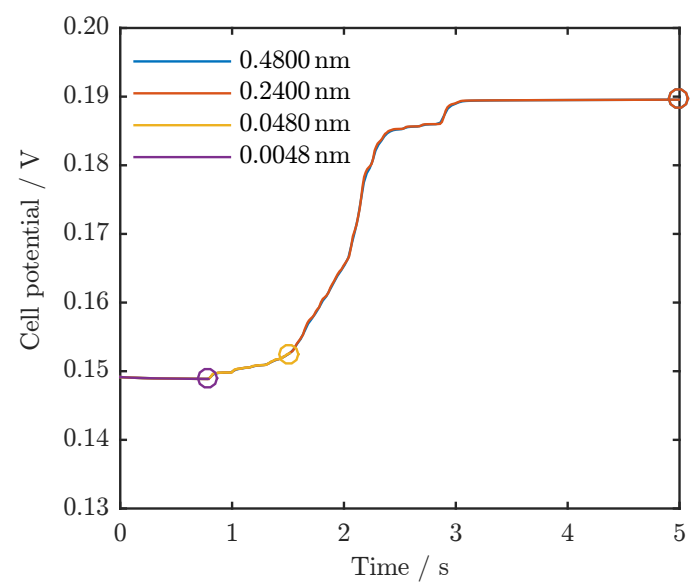

Figure 2: The influence of the prefactor on the cell potential for a stripping simulation for the $S(1 / 0 / 0)$ half-cell. The location when the simulation stopped, is marked with a colored circle. The simulation with $0.24 \mathrm{~nm}$ stops just before the $5 \mathrm{~s}$. Smaller values increase the numerical instability and hence the runtime of the simulation. Larger numerical instabilities force the solver to complete more iterations to reach a stable solution of the system.

the shape of the cell potential is not influenced by the exact value of the prefactor. Smaller prefactors force the solver to calculate more iteration steps, when the lithium phase is depleting. Each iteration step also take 


\subsection{Microstructures}

\subsubsection{Basic microstructures}

The graphite microstructure in this study is generated with the software GeoDict [25] based on literature values for the volume fractions: 0.5311 for graphite (Hitachi SMG), 0.0343 for conductive agent (Timcal Super C65) and 0.0346 for binder (Solvay Solef 5130). As particle shape for graphite a prismatic/planar polyhedron shape was selected based on SEM images from experimental cells [26]. The cathode microstructure is based on experimental data for nickel manganese cobalt oxide [27]. Binder and conductive agent were added to the electrode structures as active material, to generate a

215 "correct" pore volume. The lithium metal counter electrode is represented by a flat sheet.

The geometry of the full cell simulation is shown in Fig. 11 The microstructure of the full cell consists of $344 \times 200 \times 200$ discrete volumes (voxels), which are cubic and have a side length of $0.37 \mu \mathrm{m}$. The microstructure of the half-cell consists of the anode of the full cell ${ }^{250}$ and a flat lithium counter-electrode.

\subsubsection{Modifications for stripping simulations}

The anode structure is modified for the stripping simulations. The microstructure is partitioned into 96 volumetric sectors with a size of $30 \times 50 \times 50$ voxels: In $\mathrm{x}-$ direction (See Sec. 2.1) the microstructure is divided ${ }_{255}$ into six slabs with a thickness of 30 voxels each. These slabs are further subdivided into 16 equally sized parts, with a size of 50x50 voxels in lateral direction

The phase dedicated for an additional lithium phase is added to each of these 96 sector in two different ways: 260 A voxel of the new phase is inserted at the interface between the active material and the pore volume (electrolyte phase) by either replacing electrolyte volume or anode volume. Following this algorithm the total solid phase or the total electrolyte phase is unchanged.

One plated sector together with the other 95 unplated sectors are combined to form a modified microstructure. Therefore the 192 plated sectors result in 192 microstructures, which contain a lithium phase at different locations. In Fig. 3 one of the these modified 270 microstructures is shown, where the sector, where the lithium phase was added on, is highlighted.

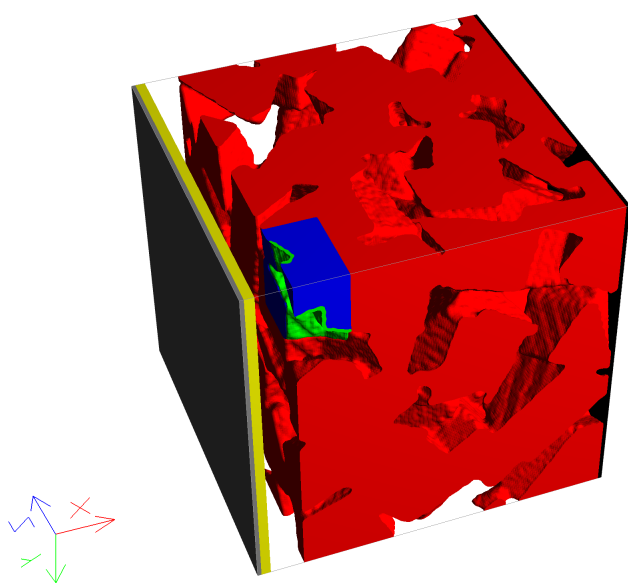

Figure 3: The modified microstructures $S(1 / 0 / 0)$ for the half-cell simulations: The anode consists of the lithium counter electrode (yellow) and the cathode of the modified graphite microstructure (Red/Blue). The sector, which has an added lithium phase (green) is highlighted in blue.

The different microstructures are identified by the location of the plated sector (coordinates of the lower left corner) and the phase (Solid or Electrolyte), which was not partially replaced by lithium. E.g. S(1/0/0) points to the microstructure, where the sector at the separatoranode interface in the lower left corner is modified by replacing electrolyte voxels with lithium. The different sectors are combinations of $x \in(1,31,61,91,121,151)$, $y \in(0,50,100,150)$ and $z \in(0,50,100,150)$.

\subsection{Summary of reactions}

The chemical intercalation is driven by the open circuit potential of the anode material underneath the plated lithium (Eq. (21)) and hence depends on the state of charge of the anode. The state of charge during the delithiation simulations is changing locally by less then $0.4 \%$. The open circuit potential $U_{0}$ of graphite at a state of charge (SoC) of $70 \%$ (initial value for stripping simulations) is nearly constant: A variation of SoC by $\pm 0.4 \%$ results in a potential difference of less then $0.17 \mathrm{mV}$. Therefore the current of the chemical intercalation can be approximated by a constant value. The resulting constant lithium intercalation shifts the point, where the plating plateau ends to earlier times. The strength of the chemical intercalation then only depends on the rate constant. Since no literature values exist for the corresponding rate constant, a correct parametrization of this constant in cooperation with specific experiments is beyond the scope of this work. For the sake 
of simplicity, the chemical intercalation reaction is neglected during the current driven stripping simulations.

In Tab. 2 the equations used for the interface currents for the different simulation setups are listed.

Table 2: List of expression used for the interface currents in the different simulations.

\begin{tabular}{|c|c|c|c|}
\hline Cell & Simulation & Interface & Equation \\
\hline \multirow[t]{2}{*}{$\mathrm{FC}$} & \multirow{2}{*}{$\begin{array}{l}\text { Plating } \\
\text { condition }\end{array}$} & Gr-El & Eq. (14) \\
\hline & & LMO-El & Eq. (14) \\
\hline \multirow[t]{4}{*}{$\mathrm{FC}$} & \multirow{4}{*}{$\begin{array}{c}\text { Stripping } \\
\text { with } \\
\text { current }\end{array}$} & Gr-El & Eq. (14) \\
\hline & & PL-El & Eq. (22) \\
\hline & & Gr-PL & neglected \\
\hline & & LMO-El & Eq. (14) \\
\hline \multirow[t]{4}{*}{$\mathrm{HC}$} & \multirow{4}{*}{$\begin{array}{c}\text { Stripping } \\
\text { with } \\
\text { current }\end{array}$} & Gr-El & Eq. (14) \\
\hline & & Gr-PL & neglected \\
\hline & & PL-El & Eq. (22) \\
\hline & & Li-El & Eq. (18) \\
\hline
\end{tabular}

FC - full-cell; HC - half-cell; Gr - Graphite; PL Plated lithium; Li - Lithium counter electrode; El - Electrolyte

\subsection{Plating condition}

It is well known, that the onset of lithium plating depends on ambient temperature and applied current. As described in Sec. 2.2.2 lithium plating is thermodynamically possible if the overpotential is less then zero. The influences on the plating condition during full cell simulations is investigated by conducting full cell simulations for various ambient temperatures and applied currents. The temperature dependence of the various material parameters are either taken directly from literature or assumed to follow the Arrhenius law (Eq. 25) with ${ }^{300}$ an estimated activation energy.

$$
f(T)=f_{0} \cdot \exp \left(-\frac{E_{\text {Activation }}}{R \cdot T}\right)
$$

For some parameters, the temperature influence may be neglected. In Tab. 4 the material parameters used for our simulations are listed.

\subsection{Influence of a microscopic resolved metallic ${ }_{310}$ lithium phase}

In Fig. 4 experimental and simulated stripping cell potentials are shown. The experimental data are extracted from figure 7 in [6] and show the constant current discharge of a cell at $-20{ }^{\circ} \mathrm{C}$ (low temperature - 315 LT) after charging at room temperature (RT)(blue) and after charging at $-20^{\circ} \mathrm{C}$ (red). The comparison of the curves in Fig. 4 a) reveals a increased cell potential for the discharge after LT charging. The cell potential of a
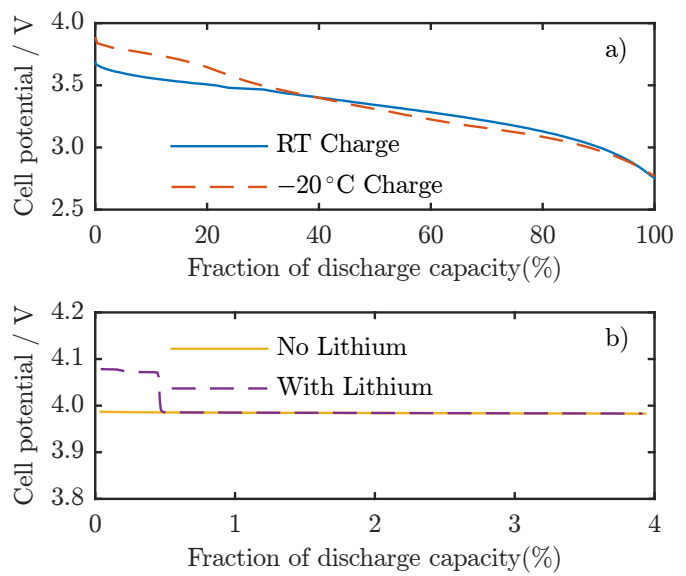

Figure 4: a) Experimental cell potentials (data extracted from [6]) during discharge at $-20^{\circ} \mathrm{C}$ after charging at room temperature (blue) and at $-20^{\circ} \mathrm{C}$ (red). A potential plateau during discharge after charging at low temperatures can be seen (Figure 7 in $[6]$ ). b) Cell potential of full cell simulation with and without a plated lithium phase on top of the anode at the anode-separator interface.

290

full cell simulation with and without plated lithium at the anode-separator-interfaces (Fig. 4 p)) show a similar behavior as the experimental one. The differences in plateau height and duration between experimental and simulated are due to the lower fraction of surface covered by plated lithium and the smaller amount of plated lithium in the simulations. The cathode material of the experiments is $\mathrm{NCO}\left(\mathrm{LiNi}_{0.8} \mathrm{Co}_{0.2} \mathrm{O}_{2}\right)$ and for the simulation LMO, thus the level of the initial cell potentials differ. These plateaus in the discharge cell potential are present, if the battery contains plated lithium. The stripping plateau in the simulation is purely a result of the additional lithium phase on the anode side. To exclude effects of the cathode all further stripping simulations are done in half-cell simulation for the anode microstructure.

To investigate, how the location of plated lithium impacts the cell performance, the microstructure was partitioned as described in Sec.3.2.2 The amount of plated lithium is initialized to a thickness of roughly $50 \mathrm{~nm}$. The total amount of plated lithium varies between the different sectors (difference between minimal and maximal amount is $68 \%$ ), since the size of electrode surface, which the additional phase was added on, in the different sectors differs.

The stripping simulations are conducted for three 
different applied current densities, which are listed in Tab. 3. In a full-cell plated lithium is stripped during discharge, where the anode gets delithiated. In a halfcell stripping will take place during charge or delithiation of the graphite, thus the half-cell are charged.

Table 3: The three different applied current density magnitudes used in the stripping simulations. The current density is with regard to the area of the current collector.

\begin{tabular}{ccc}
\hline \hline Name & Value & C-Rate \\
\hline Current 1 & $0.0020 \mathrm{~A} / \mathrm{cm}^{2}$ & $1 \mathrm{C}$ \\
Current 2 & $0.0010 \mathrm{~A} / \mathrm{cm}^{2}$ & $\mathrm{C} / 2$ \\
Current 3 & $0.0005 \mathrm{~A} / \mathrm{cm}^{2}$ & $\mathrm{C} / 4$ \\
\hline \hline
\end{tabular}

\subsection{Parameters and simulation modes}

The half-cell simulation uses the porous graphite electrode with a flat lithium counter-electrode. The full cell simulations use the porous graphite microstructure ${ }_{36}$ as negative electrode and the porous LMO microstructure as positive electrode. The separator is represented by an effective porous medium between the two electrodes. In Fig. 1 the geometry of the full cell is shown. The different material parameters of the various phases are listed in Tab. 4. In all simulation we used LiPF6 in PC/EC/DMC as electrolyte.

In Tab. 5 the initial parameters for the different simulations are listed. The full cell simulations are during charging and the half-cell simulations are during discharging.

Table 5: Initial values of the concentration and potentials for the different simulations.

\begin{tabular}{lccc}
\hline \hline Parameter & \multicolumn{2}{c}{ Value } & Unit \\
& Full Cell & Half-cell & \\
\hline$c_{\text {Graphite }}^{0}$ & 1.3195 & 18.473 & $\mathrm{kmol} / \mathrm{m}^{3}$ \\
$c_{\mathrm{LMO}}^{0}$ & 20.574 & - & $\mathrm{kmol} / \mathrm{m}^{3}$ \\
$c_{\mathrm{El}}^{0}$ & \multicolumn{2}{c}{1.200} & $\mathrm{~mol} / \mathrm{l}$ \\
$\Phi_{\text {Graphite }}^{0}$ & \multicolumn{2}{c}{$U_{0, \mathrm{a}}\left(c_{\mathrm{a}}^{0}\right)$} & $\mathrm{V}$ \\
$\Phi_{\mathrm{LMO}}^{0}$ & $U_{0, \mathrm{c}}\left(c_{\mathrm{c}}^{0}\right)$ & - & $\mathrm{V}$ \\
$\Phi_{\mathrm{Lithium}}^{0}$ & - & 0 & $\mathrm{~V}$ \\
$\varphi_{\mathrm{El}}^{0}$ & 0 & 0 & $\mathrm{~V}$ \\
\hline \hline
\end{tabular}

\section{Results and discussion}

\subsection{Plating condition}

We investigated for different charging protocols and in the anode the plating condition was first satisfied. For a battery with a base surface of $74 \mu \mathrm{m} \times 74 \mu \mathrm{m}$ $(200 \times 200$ voxels $)$, we consistently found for all conditions, that the plating condition is first reached at the anter interface of the anode. Once the condition is fulfilled at this interface, the plating boundary moves front-like towards the current collector, creating a domain between separator and current collector with increasing probability of plating, since the potential keeps droping below zero Volt against $\mathrm{Li}_{\mathrm{Li}} \mathrm{Li}^{+}$. The most negative value always are reached at the graphite-separator interface. In lateral direction no inhomogeneities in the plating condition could be seen, which can be explained by the statistical homogeneity of the investigated microstructure. If this homogeneity is violated, plated lithium films which are not homogeneously distributed at the anode-separator interface may be observed e.g. for large area cells (pouch cells). The simulation of larger pouch cells with structural inhomogeneities within the anode-separator plane was beyond the scope of our paper.

For the full cell the cell potential for different ambient temperatures and applied currents are shown in Fig. 5. The plateau exhibited by all simulations is cre-

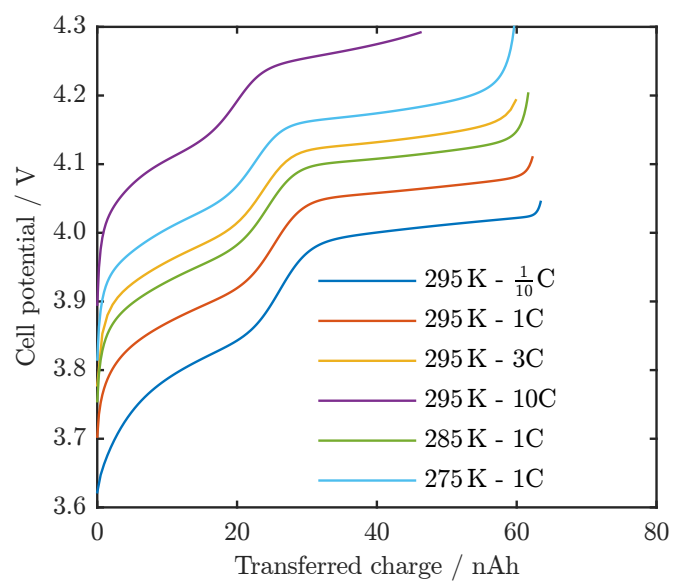

Figure 5: Cell potential of full cell simulation for different applied currents and ambient temperatures.

ated by the structure of the open circuit $U_{0}^{\mathrm{LMO}}$ of the cathode material. The different "cut-off"-potentials are results from the simulations. If a solid part of the cell reaches the maximal allowed concentration, the simulation stops. Since this is heavily influenced by applied current and the solid diffusion constant, which is a function of temperature, the final potential and transferred charge varies between the different operation modes. 
Table 4: Material and kinetic parameters used for the simulations.

\begin{tabular}{|c|c|c|c|c|c|c|c|c|c|}
\hline & \multicolumn{2}{|c|}{ Graphite } & \multicolumn{2}{|c|}{ LMO } & \multicolumn{2}{|c|}{ Li metal } & \multicolumn{3}{|c|}{ LiPF6 in PC/EC/DMC } \\
\hline Parameter/Unit & Value & Ref & Value & Ref & Value & Ref & $\mathrm{P} / \mathrm{U}$ & Value & Ref \\
\hline$c_{\max } / \mathrm{kmol} / \mathrm{m}^{3}$ & 26.39 & [28] & 23.671 & [29] & & & $t_{+} / \quad-$ & 0.399 & [30] \\
\hline$\sigma / \mathrm{S} / \mathrm{cm}$ & 10 & {$[28,31,32]^{a b}$} & 0.38 & {$[28]^{a}$} & & & $f_{ \pm} / \quad-$ & Eq. 6 in & [30] \\
\hline D $/ 10^{-10} \frac{\mathrm{cm}^{2}}{s}$ & 3.9 & {$[28,33]$} & 10 & {$[28,32]$} & & & $\kappa / \mathrm{S} / \mathrm{cm}^{2}$ & Eq. 17 in & [30] \\
\hline$U_{0} / \mathrm{V}$ & $0.63+\ldots$ & [34] & $4.06+\ldots$ & [29, 35] & & & $\mathrm{D} / \mathrm{cm}^{2} / \mathrm{s}$ & Eq. 14 in & [30] \\
\hline$i_{\text {Inter }}^{00} / \frac{\mathrm{Acm}^{5 / 2}}{\mathrm{mil}^{3 / 2}}$ & 0.088 & {$[32]^{c}$} & 0.8 & {$[32]^{c}$} & & & & & \\
\hline $\begin{array}{l}t_{\text {Plat }} \mathrm{mol}^{1 / 2 \mathrm{~cm}^{1 / 2}} \\
E_{a c t}^{C} / \mathrm{kJ} / \mathrm{mol} /\end{array}$ & 53.1 & [33] & 53.1 & $d$ & 0.0364 & [36] & & & \\
\hline$E_{a c t}^{i_{00}} / \mathrm{kJ} / \mathrm{mol}$ & 68 & [37] & 50 & {$[37]^{e}$} & 0 & $f$ & & & \\
\hline$E_{a c t}^{\sigma} / \mathrm{kJ} / \mathrm{mol}$ & 0 & $a$ & 0 & $a$ & & & & & \\
\hline
\end{tabular}

$a^{a}$ - Conductivity to large to have effect on $\Phi$, therefore assumed to be constant in $\mathrm{T}^{b}{ }^{b}$ - Literature values vary, this value selected; ${ }^{c}$ - estimated from literature for initial conditions; ${ }^{d}$ - no literature value, assumed since cathode does not strongly influence plating condition; ${ }^{e}$ - no literature value, approximated out of LFP and NCA; ${ }^{f}$ - no temperature dependence needed, since stripping simulation only at one temperature

An increase of the applied current, leads to a higher 335 overall cell potential. The higher the current, the faster the maximal concentration of the negative electrode surface is reached at which locally the OCP diverges, leading to the end of the simulations. A decrease of the ambient temperature has a similar effect on the cell potential as an increase of the applied current. The charging with $3 \mathrm{C}$ at $295 \mathrm{~K}$ shows nearly the same cell potential as the charging with $1 \mathrm{C}$ at $285 \mathrm{~K}$.

For the different operating conditions, the value of the plating condition at the graphite-separator interface 395 is shown in Fig.6.

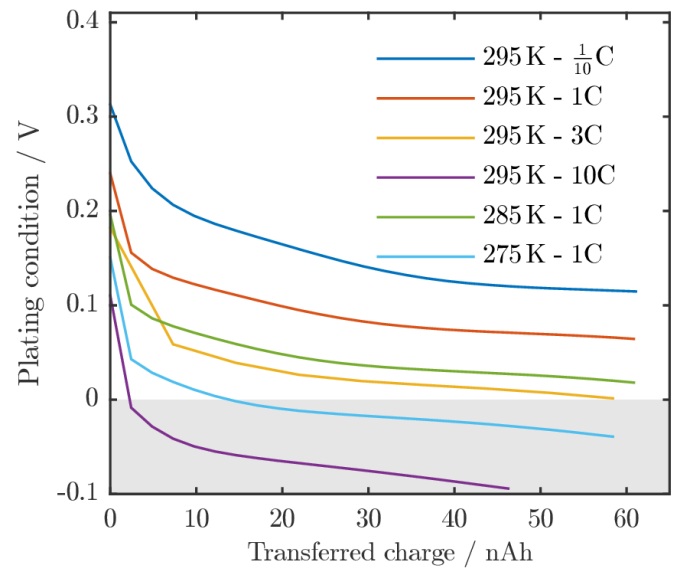

Figure 6: The plating condition for different operating conditions at the graphite-separator interface.
As expected, the plating condition is most probably fulfilled for charging at low temperatures or with high currents. With the assumption, that plating starts, as soon as the plating condition is fulfilled and keeps going afterward, a negative plating overpotential $\eta_{\text {plating }}$ early during charging should lead to an larger amount of plated lithium. How plating changes the local potential inside the battery is experimentally difficult to measure. Experiments with three-electrode-setups are able to extract the half-cell potential of the complete negative electrode, but give no exact information for the different parts of an electrode [38, 39]. The simulations for various temperatures and applied currents show, that the overpotential is negative after a short time for high current (10C) and low temperatures (275 K). These simu400 lation results coincide with experimental observations, that lithium is plating for low temperatures and high currents for the simulated materials [39]. In [16] experimental and simulation studies were conducted using the porous-electrode theory. In our work the plating 405 condition is identified as the point where the overpotential for lithium deposition becomes negative, i.e. thermodynamically favourable. It is equivalent to the point where the difference in the electrochemical potential between lithium and electrolyte (Eq. (17)) becomes negby the point, where the electrical potential of the solid anode phase reaches negative values. This is not the same as the electrochemical potential becoming negative as in our criterion (see 19). In addition the definition of the OCP of lithiated graphite in[16] allows for negative values for concentrations below the maximal 
concentration, which is actually not possible thermodynamically. Our equilibrium OCP is strictly larger zero for all concentrations of lithium. Nevertheless, qualitaconclusions for the temperature and current dependence of plating have been reached in [16].

\subsection{Lithium stripping/dissolution}

Due to the large amount of simulations in Fig. 7 the cell potential during delithiation is only shown for the sectors along the $\mathrm{x}$-axis in the corner of $\mathrm{y}=0$ and $\mathrm{z}=0$. 455 Other cross section behave similarly. The cell potentials

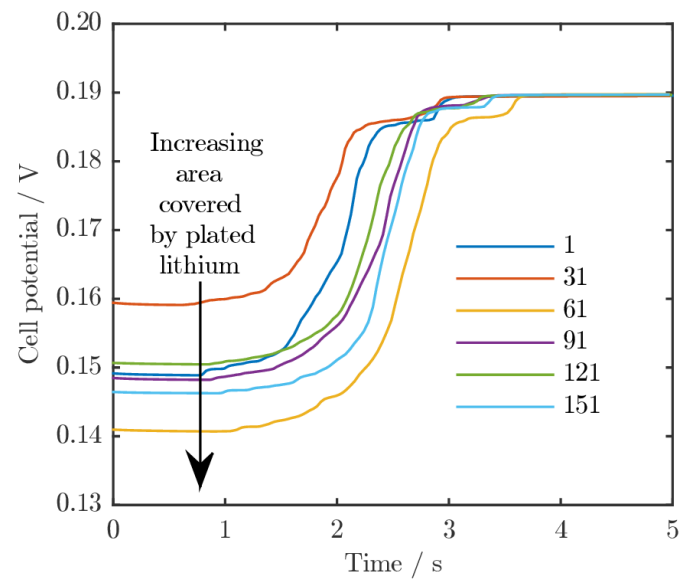

Figure 7: Cell potential during constant current charge with lithium stripping for the sectors along the $\mathrm{x}$-axis with $\mathrm{y}=0 / \mathrm{z}=0$. The $\mathrm{x}$ coordinates of the plated sector for the shown simulations are given in the figure legend. The arrow indicates the direction of increasing surface area covered by the plated lithium phase.

exhibit a plateau at the beginning of delithiation, followed by the first slow and then faster increase in potential. The final potential value is the same for all simulations. The initial plateau is due to the stripping process, ${ }^{46}$ which proceeds at constant potential. When nearly all lithium is consumed, a strong increase in the cell potential can be seen. The smaller steps in the cell potentials close to the final plateau are a result of the tensorial discretization and would turn into a smooth transition for 470 surface adapted meshes. The apparent plateau at about $0.19 \mathrm{~V}$ is the cell potential of the supporting graphite for a state of charge of $70 \%$ with the overpotential of a charge at constant current. The actual change in the poFor longer times, the cell potential will increase with the shape of the open circuit potential $U_{0}^{\text {Graphite }}$ (See Tab. 4), as usual.

For the simplification of the analysis, the cell poten- utive linear fits (See Appendix A). The intersections of the three lines are used for the estimation of the potential jump between the stripping plateau and the graphite potential, the duration of the stripping plateau 450 amount of charge transferred during the plateau. The mean of the potential jump for all 192 simulations is $\Delta \bar{U}_{\text {plateau }}=44.5 \mathrm{mV}$ with an standard deviation of $5.6 \mathrm{mV}$. The stripping time is $\bar{t}_{\text {stripping }}=2.48 \mathrm{~s}$ with an standard deviation of $0.25 \mathrm{~s}$.

In Fig. 8 the concentration in the electrolyte for three different plated sectors at $0.6 \mathrm{~s}$ and $5 \mathrm{~s}$ are shown. During the stripping of the plated lithium a concentra-

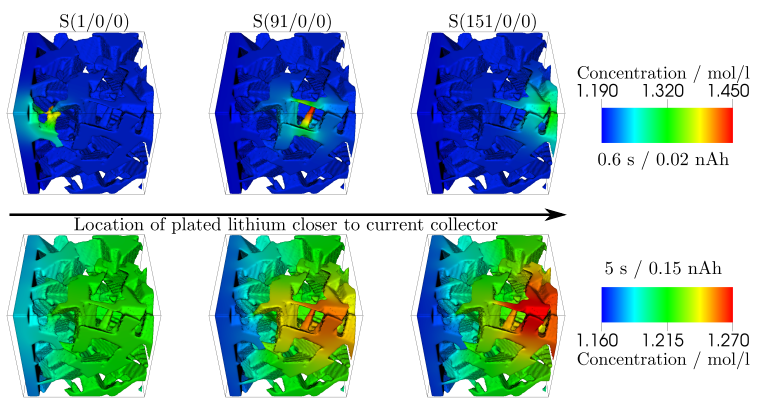

Figure 8: Lithium concentration in the electrolyte for three different plated sectors (1, 91 and 151 from Fig. 7) at $0.6 \mathrm{~s}$ (upper row) and $5 \mathrm{~s}$ (lower row). During lithium stripping a maximum close to the plated lithium is found. At the end of the simulation these maxima are less prominent.

tion maximum around the sector is visible. The concentration maxima are spatially localized. The height of the concentration maximum depends on the pore connectivity close to the sector: a smaller pore, as seen for the sector $\mathrm{S} / 91 / 0 / 0$ ), results in a higher concentration. After the plated lithium is completely stripped, the concentration maxima slowly vanish due to the lithium diffusion. The concentration profile of a plated cell will then relax to the profile of a unplated cell, where the lowest concentration is close to the anode current collector and highest concentration close to the cathode current collector. Cells with a plated sector close to the anode current collector take more time to reach the profile of a unplated cell.

The zoning behavior of the concentration maxima can not be resolved in a 1D+1D-Newman-type simulation, since the concentration in the electrolyte in the lateral direction is not resolved.

The electrochemical potential of lithium ions in the electrolyte also inhibit a maximum close to plated lithium. This maximum, similar to the concentration one, dissipates after all plated lithium is stripped.

All microstructures used for stripping simulation 
have the same thickness of lithium added. The surface area onto which the lithium is added differs between the modified microstructures and thus the total amount of 510 Lithium added. The initial level and the duration of the potential plateau depend on the size of plated surface and the lithium thickness. Hence the cell potentials shown in Fig. 7 show some variation in initial level and stripping duration. In the following section the influ- 515 ence of the distance between plated lithium and current collector, surface area and amount of lithium on the potential plateau is investigated in more detail.

\subsubsection{Influences on value and length of potential plateau \\ Distance to current collector}

The different modified microstructures do not have the same value of surface area covered by the plated lithium phase. To check how the distance between the plated lithium and the current collector influences the potential plateau, the simulation results are grouped by similar surface areas. The cell potentials for microstructures with a plated surface area between $8.50 \cdot 10^{-6} \mathrm{~cm}^{2}$ and $8.75 \cdot 10^{-6} \mathrm{~cm}^{2}$ are sorted by their respective distance to the current collector and are shown in Fig.9. The dis-

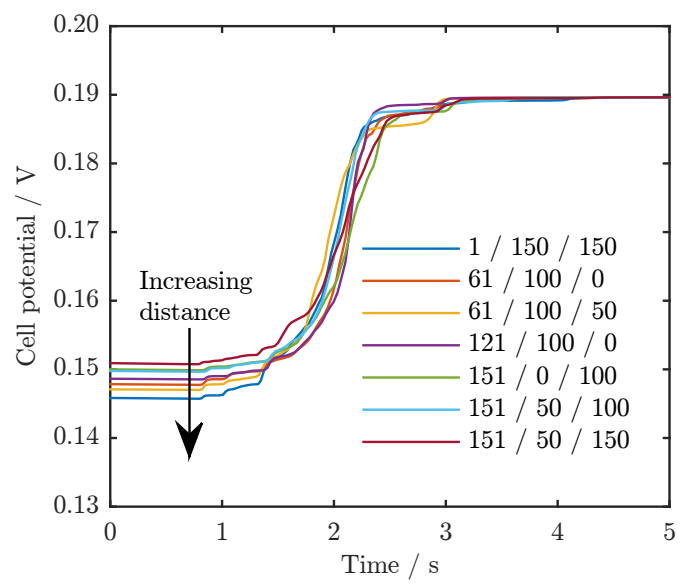

Figure 9: Cell potential of half-cell during constant current charge with lithium stripping for microstructure with a lithium covered surface of $8.50 \cdot 10^{-6} \mathrm{~cm}^{2}-8.75 \cdot 10^{-6} \mathrm{~cm}^{2}$ ). The coordinates of the plated sector for the shown simulations are given in the figure legend. The arrow indicates the direction of increasing distance to the current collector.

tance between the plated lithium and the current collector has only a small influence on the plateau height and 555 an even smaller impact on the transferred charge. The change in the plateau height for the selected simulation is about $5 \mathrm{mV}$ for a distance of roughly $66 \mu \mathrm{m}$. The electrolyte resistance alone is not responsible for this small distance dependence: For a constant electrolyte concentration (e.g.: at the start of delithiation, when the concentration had no time to respond), the electrochemical potential in the electrolyte decreases by around $1 \mathrm{mV}$ over a distance of $70 \mu \mathrm{m}$. The difference in cell potential for increasing distance to the graphite current collector is in the order for $5 \mathrm{mV}$.

\section{Covered surface area}

In Fig. 7 the effect of the surface area is indicated by an arrow. The potential jump increases with the covered surface area i.e. the stripping plateau is lowered towards the $\mathrm{Li} / \mathrm{Li}^{+}$redox potential. The covered surface area for the simulation at $\mathrm{x}=31$ and $\mathrm{x}=61$ as seen in Fig. 6 is $5.8 \cdot 10^{-6} \mathrm{~cm}^{2}$ and $12.7 \cdot 10^{-6} \mathrm{~cm}^{2}$ respectively and the initial potential level is $159 \mathrm{mV}$ (potential jump of $30 \mathrm{mV}$ ) and $141 \mathrm{mV}$, respectively (potential jump of $49 \mathrm{mV}$ ). The covered surfaces area is increased constitutes by $112 \%$, which results in an lowering of the plateau level by $63 \%$. A higher coverage of the surface area with plated lithium lowers the initial cell potential further. Should the complete area of the graphite be covered by lithium, an effective lithium/lithium-cell is created. The difference between the stripping plateau and $\mathrm{Li} / \mathrm{Li}^{+}$potential is then given by the overpotential due to the applied current only.

The lowest surface area coverage is $5.8 \cdot 10^{-6} \mathrm{~cm}^{2}$ and the largest is $12.7 \cdot 10^{-6} \mathrm{~cm}^{2}$. These correspond to a fraction of the active surface area covered by plated lithium of $0.6 \%$ and $1.3 \%$. In comparison with the distance from the current collector (see previous section), the coverage area has a larger impact on the change in plateau height. For the simulations shown in Fig. 7t the plateau value changes by $19 \mathrm{mV}$.

The amount of covered surface area also influences the total charge to be transfered until the lithium is dissolved. As expected, an increase of the covered surface area leads to an increase in transferred charge. But this is not only a result of the different volume of metallic lithium. The transferred charge will also depend on the ratio of the intercalation exchange current amplitude and the plating exchange current amplitude as well as on the applied current and details of the microstructure, as discussed in more detail in the next section.

\subsubsection{Interface currents during stripping}

The simulation of a 3D lithium-ion battery allows to investigate the distribution of currents at the interfaces during the stripping. In Fig. 10 the stripping current and the graphite intercalation current are shown. They are 
normalized to the applied current to allow for a direct comparison.

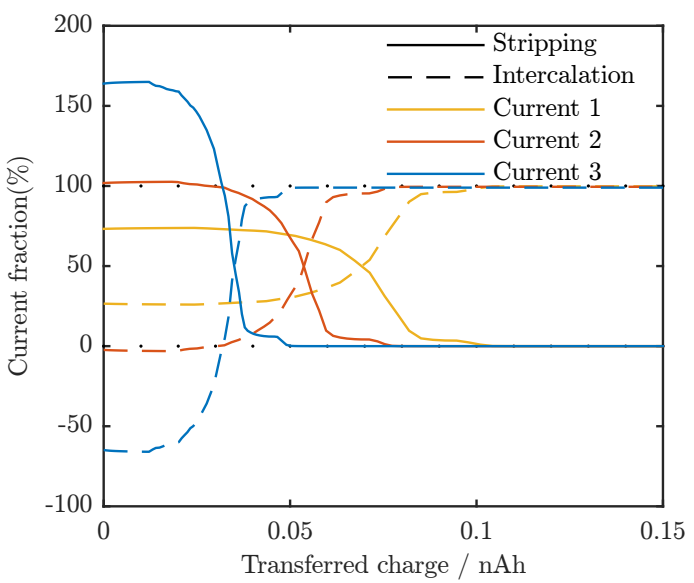

Figure 10: The distribution of the total current on the two reactions for the three different applied currents (See Tab. 3) against the transferred charge. Shown for lithium in sector $\mathrm{S}(91 / 0 / 0)$.

The first interesting observation is, that the net charge transferred during the stripping plateau is smaller for smaller applied currents. This surprising finding is due to a subtle competition between local oxidation of plated lithium and reduction of lithium ions during inercalation into graphite. It turns out that especially at low current the oxidative stripping of the lithium film leads to an effective surplus of lithium ions in the adjacent electrolyte. These lithium ions can then contribute to the intercalation current into the graphite. Thereor, the fraction of charge carried by the total interface current between the graphite and the electrolyte can either be positive, zero or negative. A positive interface current is identical to stripping or deintercalation, while a negative interface current is only observed for the graphite part of the interface and is due to intercalation. Hence, for the higher current (Current 1) the lithium de-intercalates from the graphite and for the lowest current (Current 3) lithium intercalates into the graphite. The change in lithium concentration in the graphite can be seen in Fig. 11 It is clearly visible, that for the lowest current the concentration of lithium ions in the graphite is first increasing before net deintercalation sets in. Only at larger currents, deintercalation i.e. monotonous decrease of lithium is observed for all

In Fig. 12 the sign of the interface currents for the three different applied currents in the 3D structure are shown. Only the voxels at the surface of the graphite

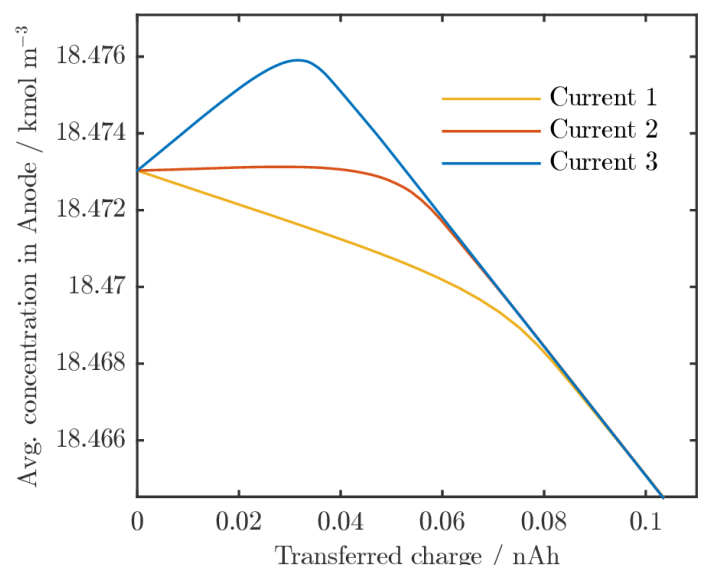

Figure 11: The average concentration in graphite for plated lithium in the sector $(91 / 0 / 0)$ for three different applied currents.

and plated lithium are plotted. Other phases (counter electrode, electrolyte,...) are transparent. Areas with lithiation or deliathiation (Eq. (I-A) ), plating or stirpping (Eq. (S1)) are shown color coded.

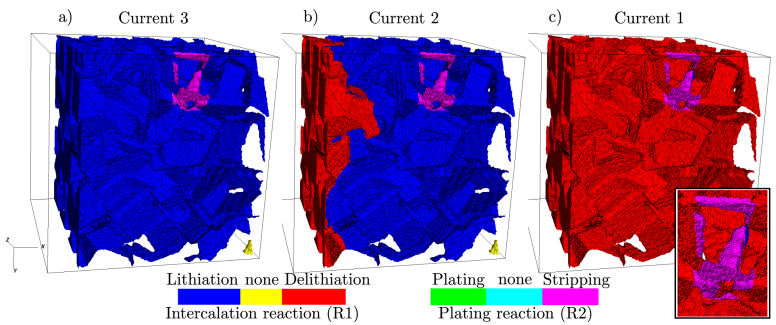

Figure 12: The sign of the interface currents for three different applied currents (See Tab. 3 in 3D representation during delithiation at $0.02 n A h$. For the electrolyte-graphite interface blue indicates intercalation and red delithiation and for the electrolyte-plated lithiuminterface green represents plating and pink stripping. For all three currents, the plated lithium is stripped, while the graphite either intercalates or deintercalates lithium, depending on the current. Shown for lithium in sector $S(91 / 0 / 0)$.

The plated sector is visible as the pink sector for all currents, which indicates, that lithium is getting stripped. It can be clearly seen, that for the smallest current (Fig. 12]a) ), only the plated lithium sector dissolves lithium into the electrolyte, while lithium is intercalated from the electrolyte into the graphite. The interface current for the second current (Fig. 12 b) ) indicates, that the graphite only deintercalates lithium at the separatorelectrode-interface. For the highest current (Fig. 12 c) ) the graphite shows nearly everywhere delithiation, except for a small portion close to the plated lithium sector (shown in inset in lower right corner in Fig. 12). 
Noteworthy is the small yellow area in the lower right corner of first two plot (inset covers it in plot c) ) in Fig. 12. This area indicates a unconnected electrolyte pore and therefore an inactive interface.

\subsubsection{Estimation of amount of plated lithium}

The method of differential capacity, as described in [9], is a promising method to estimate the amount of lithium plated inside a battery by only measuring the discharge cell potential. The interpretation of the potential drop duration as a marker for the amount of lated lithium is supported by neutron diffraction measurements [12]. By calculating the derivative of the cell potential with respect to the transferred current $Q$ and selecting the position of highest change, the amount of lithium is estimated. Our 3D resolved simulations alow for a more detailed investigation of the potential and limitations of the proposed method. In Fig. 13 the differential capacity is shown for the simulations in Fig. 10 at the three different applied currents. The total amount of charged transferred by converting the lithium layer into lithium ions is marked as solid black line and the estimated amounts are shown as dashed, dotted or colored solid line.

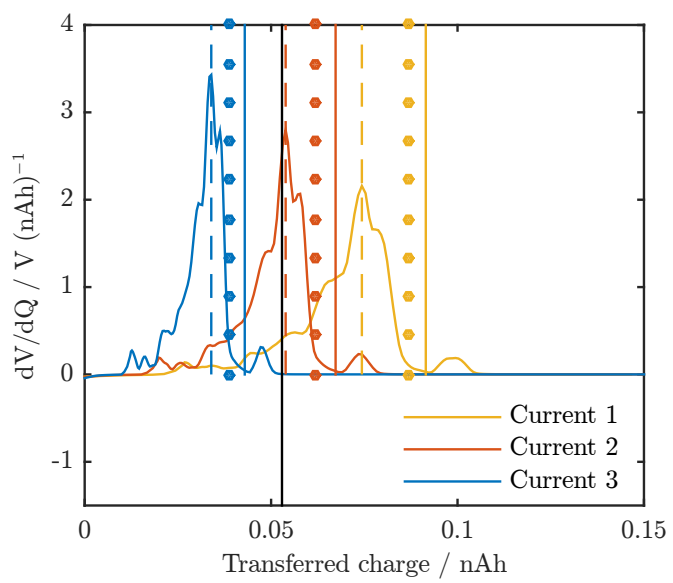

Figure 13: The differential capacity calculated for the three different 665 currents.

The dashed line shows the estimation done by performing the differential capacity method on the simulation results, the dotted line is the amount of lithium 670 obtained via point 2 of the line fits (See Appendix A) and the solid colored line represents the position, when the average thickness of the plated lithium in the system is less then $0.48 \mathrm{~nm}$. For a given applied current, methods. Also a dependency on the applied current is clearly recognizable. For a certain current it can happen that the estimated amount of a method is the same as the exact amount of lithium, however in experiments the applied current the amount of plated lithium can be overestimated or underestimated. I.e., with the differential capacity method only a rough approximation of the plated lithium can be obtained. In Tab. 6 the deviation of the estimated amount of lithium from the real amount of lithium for the three different analysis methods is shown.

Table 6: Difference between correct amount of lithium and estimated.

\begin{tabular}{cccc}
\hline \hline Current & \multicolumn{3}{c}{ Error of method in \% } \\
& $\begin{array}{c}\text { dVdQ } \\
\text { (dashed) }\end{array}$ & $\begin{array}{c}\text { Fits } \\
\text { (dotted) }\end{array}$ & $\begin{array}{c}\text { avg. thickness } \\
\text { (solid) }\end{array}$ \\
\hline Current 1 & 36 & 26 & 19 \\
Current 2 & -2 & -17 & -27 \\
Current 3 & -41 & -64 & -73 \\
\hline \hline
\end{tabular}

\subsubsection{Influence of chemical intercalation}

All previous discussion are done without the chem650 intercalation enabled. In this section we want to discuss, how the chemical intercalation would effect the cell potential during stripping. As described in Sec. 3.3. the chemical intercalation does only depend on the local lithium concentration adjacent to the plated region and therefore be estimated by a constant intercalation current, which is slaved by the slow change of concentration. This approximately constant current will lead to a faster stripping process and thus an earlier end of the stripping plateau. The exact shift depends highly on the 660 rate constant of the chemical intercalation (S2) and the thickness of the plated lithium. If the mass transfer due to the chemical intercalation has the same amplitude as the stripping reaction into the electrolyte, the duration of the stripping plateau will be halved. In general, if both reaction mechanisms are active, the strength of the current dependency depends on which pathway ( (S1) and (S2) ) strips the most plated lithium. For the case, that the electrochemical stripping reaction only dissolves a very small fraction of plated lithium compared to the chemical intercalation, the current dependency will become negligible. Since we are not aware of any literature values for the chemical intercalation rate, it was not included in our simulations. Only extensive parameter studies and dedicated experiments can clarify, which reaction mechanism is dominant and what the reasons are 
for this. In the scope of this work, these studies were not possible. In a future publication, the distribution of the plating mass flux on the different possible reaction pathways will be investigated in more detail.

\section{Conclusion}

We investigated for the first time on microstructure scale the occurrence of plating and the influence of plated lithium on the charge - potential characteristics depending on the position of the plated lithium in the electrode microstructure. Full cell simulations showed in agreement with experimental results [9, 11] that the plating reaction most probably is initiated at the anode- 740 separator-interface and that the first occurrence is earlier for higher currents or lower ambient temperature. The well-known plateau during discharge of a plated lithium ion cell is also observed in our simulations and can, as 745 expected, be attributed to the reversible stripping of the plated lithium. Unexpectedly, the amount of transferred charge during stripping depends not only on the total amount of plated lithium but also on the applied current. This phenomena was explained by the occurrence of local short circuit like currents, which are caused by stripping induced intercalation of lithium ions in graphite during discharge in half-cells or charge in full cells.

Experimentally, the method of differential voltammetry [9] is used to estimated the amount of plated lithium from the amount of transferred charge. Partially due to the induction of the observed local short circuits, 760 the relation between charge transfer and plated lithium is not as unique as expected. If we apply differential voltammetry to our simulations and compare the extracted values with the lithium amount at the beginning 765 of the simulation, we see a discrepancy. The amount of plated lithium can be underestimated or overestimated, depending on the applied current.

Also the dependence of the stripping plateau on cov- 770 ered surface area, distance from current collector and applied currents have been investigated. We find, that the amount of coverage has the dominant influence on

715 the level of the stripping plateau. Its influence on the stripping plateau is much more pronounced, than the specific place of plating within the electrode. In future investigations a detailed model for the actual plating process, which is under development, will supple- ${ }^{780}$ 720 ment our stripping simulations and will allow to study the competition of stripping and plating during cycling.

\section{Acknowledgements}

Financial support from the German Ministry of Education and Research (BMBF) in the framework
This research was supported in part by the bwHPC initiative and the bwHPC-C5 project ( funded by the Ministry of Science, Research and the Arts BadenWürttemberg (MWK) and the Germany Research Foun730 dation (DFG) ) provided through associated compute services of the JUSTUS HPC facility at the University of Ulm.

[1] Ostrower, J., Pasztor, A.. Microscopic 'dendrites' a focus in Boeing Dreamliner probe. Wall Str J 2013;URL: http://www.wsj.com/articles/ SB10001424127887324880504578298673566960476

[2] Dolle, M., Sannier, L., Beaudoin, B., Trentin, M., Tarascon, J.M.. Live Scanning Electron Microscope Observations of Dendritic Growth in Lithium/Polymer Cells. Electrochem SolidState Lett 2002;5(12):A286-A289. doi 10.1149/1.1519970

[3] Cesiulis, H., Ziomek-Moroz, M.. Electrocrystallization and electrodeposition of silver on titanium nitride. $\mathbf{J}$ Appl Electrochem 2000;30(11):1261-1268. doi 10.1023/A : 1026553712521

[4] Tarascon, J.M., Armand, M.. Issues and challenges facing rechargeable lithium batteries. Nature 2001;414(6861):359367. doi $10.1038 / 35104644$

[5] Vetter, J., Novák, P., Wagner, M., Veit, C., Möller, K.C., Besenhard, J., et al. Ageing mechanisms in lithium-ion batteries. J Power Sources 2005;147(1-2):269-281. doi $10.1016 / j$. jpowsour.2005.01.006

[6] Smart, M.C., Ratnakumar, B.V.. Effects of Electrolyte Composition on Lithium Plating in Lithium-Ion Cells. J Electrochem Soc 2011;158(4):A379-A389. doi 10.1149/1.3544439

755 [7] Legrand, N., Knosp, B., Desprez, P., Lapicque, F., Raël, S.. Physical characterization of the charging process of a Liion battery and prediction of Li plating by electrochemical modelling. J Power Sources 2014;245:208-216. doi 10.1016/j . jpowsour.2013.06.130

[8] Petzl, M., Danzer, M.A.. Nondestructive detection, characterization, and quantification of lithium plating in commercial lithium-ion batteries. J Power Sources 2014;254:80-87. doi $10.1016 / j \cdot j p o w s o u r .2013 .12 .060$

[9] Petzl, M., Kasper, M., Danzer, M.A.. Lithium plating in a commercial lithium-ion battery A low-temperature aging study. J Power Sources 2014;275:799-807. doi 10.1016/j . jpowsour.2014.11.065

[10] Gireaud, L., Grugeon, S., Laruelle, S., Yrieix, B., Tarascon, J.M.. Lithium metal stripping/plating mechanisms studies: A metallurgical approach. Electrochem commun 2006;8(10):1639-1649. doi 10.1016/j.elecom.2006.07. 037

[11] Harris, S.J., Timmons, A., Baker, D.R., Monroe, C.. Direct in situ measurements of $\mathrm{Li}$ transport in Li-ion battery negative electrodes. Chem Phys Lett 2010;485(4-6):265-274. doi 10 . 1016/j.cplett.2009.12.033

[12] Zinth, V., von Lüders, C., Hofmann, M., Hattendorff, J., Buchberger, I., Erhard, S., et al. Lithium plating in lithiumion batteries at sub-ambient temperatures investigated by in situ neutron diffraction. J Power Sources 2014;271:152-159. doi $10.1016 / j$.jpowsour .2014.07.168

[13] Newman, J., Tiedemann, W.. Porous-electrode theory with battery applications. AIChE J 1975;21(1):25-41. doi 10.1002/ aic.690210103

[14] Arora, P., Doyle, M., White, R.E.. Mathematical Modeling of the Lithium Deposition Overcharge Reaction in LithiumIon Batteries Using CarbonBased Negative Electrodes. J Electrochem Soc 1999;146(10):3543-3553. doi 10.1149/1.1392512 
[15] Tang, M., Albertus, P., Newman, J.. Two-Dimensional Modeling of Lithium Deposition during Cell Charging. J Electrochem 855 Soc 2009;156(5):A390-A399. doi 10.1149/1.3095513

[16] Tippmann, S., Walper, D., Balboa, L., Spier, B., Bessler, W.G.. Low-temperature charging of lithium-ion cells part I: Electrochemical modeling and experimental investigation of degradation behavior. J Power Sources 2014;252:305-316. 860 doi $10.1016 / \mathrm{j}$.jpowsour.2013.12.022

[17] Ecker, M., Käbitz, S., Gerschler, J., Sauer, D.. Modeling of Lithium Plating in Lithium-ion batteries. ECS Meet 2010;218:201.

[18] Krämer, Y., Birkenmaier, C., Feinauer, J., Hintennach, A., 865 Bender, C.L., Meiler, M., et al. A New Method for Quantitative Marking of Deposited Lithium by Chemical Treatment on Graphite Anodes in Lithium-Ion Cells. Chem - A Eur J 2015;21(16):6062-6065. doi 10.1002/chem. 201406606

[19] Monroe, C., Newman, J.. Dendrite Growth in Lithium/Polymer 870 Systems. J Electrochem Soc 2003;150(10):A1377-A1384. doi 10.1149/1.1606686

[20] Akolkar, R.. Mathematical model of the dendritic growth during lithium electrodeposition. J Power Sources 2013;232:23-28. doi $10.1016 / \mathrm{j}$.jpowsour.2013.01.014

[21] Latz, A., Zausch, J.. Thermodynamic consistent transport theory of Li-ion batteries. J Power Sources 2011;196(6):32963302. doi 10.1016/j.jpowsour. 2010.11.088

[22] Latz, A., Zausch, J.. Multiscale modeling of lithium ion batteries: thermal aspects. Beilstein J Nanotechnol 2015;6:987-1007. doi 10.3762/bjnano.6.102

[23] ITWM, . BEST - Battery and Electrochemistry Simulation Tool. 2014. URL: http://itwm.fraunhofer.de/BEST

[24] Tornberg, A.K., Engquist, B.. Regularization techniques for numerical approximation of PDEs with singularities. J Sci Comput 2003;19(1/3):527-552. doi 10.1023/A :1025332815267

[25] Math2Market, . GeoDict. 2015. URL: www .geodict . com

[26] Singh, M., Kaiser, J., Hahn, H.. Thick Electrodes for High Energy Lithium Ion Batteries. J Electrochem Soc 2015;162(7):A1196-A1201. doi 10.1149/2.0401507jes

[27] Ebner, M., Geldmacher, F., Marone, F., Stampanoni, M., 875 Wood, V.. X-Ray Tomography of Porous, Transition Metal Oxide Based Lithium Ion Battery Electrodes. Adv Energy Mater 2013;3(7):845-850. doi 10.1002/aenm. 201200932

[28] Doyle, M., Newman, J., Gozdz, A.S., Schmutz, C.N., Tarascon, J.M.. Comparison of Modeling Predictions with Experimental Data from Plastic Lithium Ion Cells. J Electrochem Soc 1996;143(6):1890. doi 10.1149/1.1836921

[29] Less, G.B., Seo, J.H., Han, S., Sastry, A.M., Zausch, J., Latz, A., et al. Micro-Scale Modeling of Li-Ion Batteries: Parameterization and Validation. J Electrochem Soc 2012;159(6):A697A704. doi 10.1149/2.096205jes

[30] Valoen, L.O., Reimers, J.N.. Transport Properties of LiPF[sub 6]-Based Li-Ion Battery Electrolytes. J Electrochem Soc 2005;152(5):A882-A891. doi 10.1149/1.1872737

[31] Morgan, M.. Conduction in amorphous carbon films. Thin Solid Films 1970;7(1):313-323

[32] Fuller, T.F., Doyle, M., Newman, J.. Simulation and Optimization of the Dual Lithium Ion Insertion Cell. J Electrochem Soc 1994;141(1):1-10. doi 10.1149/1.2054684

[33] Langer, J., Epp, V., Heitjans, P., Mautner, F.A., Wilkening, M.. Lithium motion in the anode material LiC6 as seen via timedomain 7Li NMR. Phys Rev B - Condens Matter Mater Phys 2013;88(9):1-9. doi 10.1103/PhysRevB.88.094304

[34] Safari, M., Delacourt, C.. Modeling of a Commercial Graphite/LiFePO4 Cell. J Electrochem Soc 2011;158(5):A562A571. doi 10.1149/1.3567007

[35] Du, W., Gupta, A., Zhang, X., Sastry, A.M., Shyy,
W.. Effect of cycling rate, particle size and transport properties on lithium-ion cathode performance. Int J Heat Mass Transf 2010;53(17-18):3552-3561. doi $10.1016 / j$. ijheatmasstransfer.2010.04.017

[36] Chandrasekaran, R., Fuller, T.F.. Analysis of the Lithium-Ion Insertion Silicon Composite Electrode/Separator/Lithium Foil Cell. J Electrochem Soc 2011;158(8):A859-A871. doi 10 . 1149/1.3589301

[37] Jow, T.R., Marx, M.B., Allen, J.L.. Distinguishing Li+ Charge Transfer Kinetics at NCAElectrolyte and GraphiteElectrolyte Interfaces, and NCAElectrolyte and LFPElectrolyte Interfaces in Li-Ion Cells. J Electrochem Soc 2012;159(5):A604-A612. doi 10.1149/2.079205jes

[38] Ratnakumar, B.V., Smart, M.C.. Lithium Plating Behavior in Lithium-Ion Cells. ECS Trans 2010;25(36):241-252. doi 10 . $1149 / 1.3393860$

[39] Zhang, S.S., Xu, K., Jow, T.R.. Study of the charging process of a LiCoO2-based Li-ion battery. J Power Sources 2006;160(2):1349-1354. doi 10.1016/j.jpowsour. 2006 . 02.087

\section{Appendix A. Simplified cell potential plateaus}

Due to the artifacts in the cell potential for the stripping simulations, the data is separated into three regimes: One representing the initial potential level, one the part of biggest change and one representing the final potential level. Into these regions straight lines (Eq. (A.1p) are fitted.

$$
g\left(t, m, t_{0}\right)=t_{0}+m \cdot t
$$

The intersections of the three lines are used for further analysis. Fig. A.1 shows the resulting fits for the stripping simulation of sector $\mathrm{S}(1 / 0 / 0)$ (Blue curve in Fig. 7).

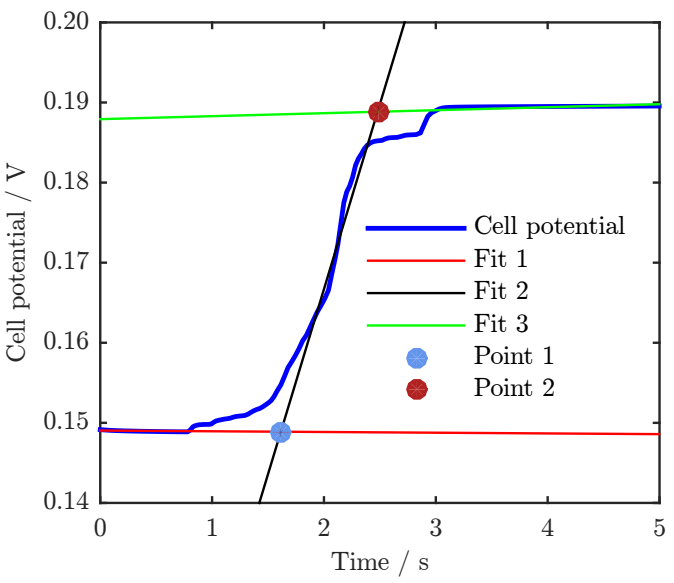

Figure A.1: Linear fits to the three regions of the stripping cell potential of sector $S(1 / 0 / 0)$. 
The intersections between the three lines are marked 880 as "point 1 " $\left(t_{1}, V_{1}\right)$ and "point 2 " $\left(t_{2}, V_{2}\right)$. The x coordinate of "point $2 " t_{2}$ is taken as the time, where we can assume, that all lithium is stripped if we had no artifacts: $t_{\text {strip }}=t_{2}$. The difference in the y coordinates between the two points $V_{2}-V_{1}$ is assumed to be the "height" of 885 the potential drop due to stripping: $\Delta U_{\text {Plateau }}=V_{2}-V_{1}$. 